Algebraic $8 \mathcal{G}$ Geometric $\mathcal{T}_{\text {opology }}$

Volume 4 (2004) 297-309

Published: 6 May 2004

ATG

\title{
Cubulating spaces with walls
}

\author{
BOGDAN NiCA
}

\begin{abstract}
We describe a correspondence between spaces with walls and $\mathrm{CAT}(0)$ cube complexes.
\end{abstract}

AMS Classification 20F65; 20E42

Keywords Space with walls, Median graph, CAT(0) cube complex

\section{Introduction}

The elegant notion of a space with walls was introduced by Haglund and Paulin 6]. Prototypical examples of spaces with walls are CAT(0) cube complexes, introduced by Gromov in [5]. The purpose of this note is to observe that every space with walls has a canonical embedding in a $\mathrm{CAT}(0)$ cube complex and, consequently, a group action on a space with walls extends naturally to a group action on a CAT(0) cube complex. The usefulness of this result is that spaces with walls are often easily identifiable by geometric reasons.

The cubulation of a space with walls, as we call it, is an abstract version of a construction introduced by Sageev [1] for the purpose of relating multiended pairs of groups to essential actions on CAT(0) cube complexes. Sageev's construction is further explored by Niblo and Roller in [7, where an essential group action on a CAT(0) cube complex is shown to imply the failure of Kazhdan's property (T) (see also [8]). Roller's detailed study [10 formulates Sageev's construction in the language of median algebras (see also [4]). Finally, a version of Sageev's construction, where a CAT(0) cube complex arises from a system of halfspaces in a complex, is considered by Niblo and Reeves [9], for Coxeter groups, and by Wise [12, for certain small cancellation groups.

Some of the papers cited above $([1],[7,[9])$ take the point of view that the primitive data for constructing a CAT(0) cube complex is a partially ordered set with an order-reversing involution, with certain discreteness and nesting assumptions à la Dunwoody, which is to become the system of halfspaces in the cube complex. However, a space with walls comes in handy when a suitable connected component needs to be specified. 
The cubulation of a space with walls comprises two steps, according to the following scheme:

space with walls $\longrightarrow$ median graph $\longrightarrow \mathrm{CAT}(0)$ cube complex

In the first step, which is our main objective, a space with walls $X$ is embedded in a median graph $\mathcal{C}^{1}(X)$, called the " 1 -cubulation of the space with walls $X$ ". The second step is based on the fact that any median graph is the 1-skeleton of a unique CAT(0) cube complex. Explicitly, the step from the median graph $\mathcal{C}^{1}(X)$ to a $\operatorname{CAT}(0)$ cube complex $\mathcal{C}(X)$ consists of "filling in" isometric copies of euclidean cubes by inductively adding an $n$-dimensional cube whenever its $(n-1)$-skeleton is present; see [11, §3], [3, §6], [10, Thm.10.3], [12, §5].

Our result is:

Theorem Let $X$ be a space with walls. There exists an injective morphism of spaces with walls

$$
X \stackrel{\sigma}{\longrightarrow} \mathcal{C}^{1}(X)
$$

where $\mathcal{C}^{1}(X)$ is a connected median graph, and $\sigma(X)$ "spans" $\mathcal{C}^{1}(X)$, in the sense that no proper subgraph of $\mathcal{C}^{1}(X)$ containing $\sigma(X)$ is median.

Spaces with walls and their morphisms are defined in Section 2. Median graphs are defined in Section 3, where we prove that they are spaces with walls. The main construction is presented in Section 4, where we also show that any group action on a space with walls has a unique extension to a group action on its 1 -cubulation.

Finally, we would like to draw the reader's attention to a different account of the cubulation procedure, described independently by Chatterji and Niblo [2]

Acknowledgements I am grateful to Dani Wise for suggesting the problem, as well as for constructive comments that have improved the content of this paper. This work has been supported by NSERC.

\section{Spaces with walls}

We recall the definition of a space with walls. We also introduce the natural notion of morphism of spaces with walls. 
Definition 2.1 Let $X$ be a set. A wall in $X$ is a partition of $X$ into 2 subsets called halfspaces. We say that $X$ is a space with walls if $X$ is endowed with a collection of walls, containing the trivial wall $\{\emptyset, X\}$, and so that any two distinct points are separated by a finite, non-zero number of walls. Note that a wall separates two distinct points $x, y \in X$ if $x$ belongs to one of the halfspaces determined by the wall, while $y$ belongs to the other halfspace.

A morphism of spaces with walls is a map $f: X \rightarrow X^{\prime}$ between spaces with walls with the property that $f^{-1}\left(A^{\prime}\right)$ is a halfspace of $X$ for each halfspace $A^{\prime}$ of $X^{\prime}$.

A minor difference between the original definition from [6] and the definition given above is that we insist on the presence of the trivial wall. This modification is needed for a morphism of spaces with walls to be well-defined. Another reason is that halfspaces arise naturally in the presence of an underlying convexity structure: a halfspace is a convex set whose complement is convex. In such a context, the trivial wall is always present.

A space with walls $X$ becomes a metric space by defining the distance between two points to be the number of walls separating them: $d_{w}(x, y)=|W(x, y)|$, where $W(x, y)$ denotes the set of walls separating $x$ and $y$. For the triangle inequality observe that, given $x, y, z$, a wall separating $x$ and $y$ has to separate either $x$ from $z$, or $z$ from $y$, i.e., $W(x, y) \subseteq W(x, z) \cup W(z, y)$. We obtain: $d_{w}(x, y)=|W(x, y)| \leq|W(x, z)|+|W(z, y)|=d_{w}(x, z)+d_{w}(z, y)$. We call $d_{w}$ the wall metric on $X$.

A group acts on a space with walls $X$ by permuting the walls. Consequently, it acts by isometries on $\left(X, d_{w}\right)$.

\section{$3 \quad$ Median graphs}

Median graphs are well documented in graph-theoretic literature. See [3, §4] for a list of papers on median graphs. Unless otherwise specified, graphs are henceforth assumed to be connected, simplicial, in the sense that they have no loops or multiple edges, and equipped with the path metric. The geodesic interval $[x, y]$ determined by the vertices $x$ and $y$ is the collection of vertices lying on a shortest path from $x$ to $y$.

Definition 3.1 A graph is median if, for each triple of vertices $x, y, z$, the geodesic intervals $[x, y],[y, z],[z, x]$ have a unique common point. 
Trees are elementary examples of median graphs. The 1-skeleton of the square tiling of the plane is median whereas the 1-skeletons of the hexagonal and triangular tilings are not.

Lemma 3.2 In a median graph the geodesic intervals are finite.

Proof Induction on the distance between vertices. Consider two vertices $x, y$ and let $Z$ denote the collection of those neighbors of $y$ that are in $[x, y]$. As $[x, y] \backslash\{y\} \subseteq \cup_{z \in Z}[x, z]$ with each $[x, z]$ finite by the induction hypothesis, it suffices to show that $Z$ is finite.

Nodes in $Z$ are pairwise distance 2 apart. Fix $a \in Z$ and let $m(z)=m(x, a, z)$ for every $z \in Z$. See Figure 1

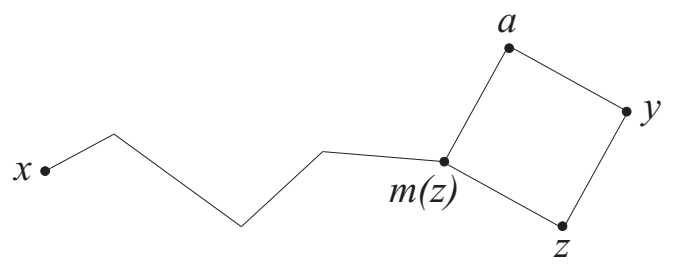

Figure 1: Finiteness of geodesic intervals in a median graph

The mapping $z \mapsto m(z)$ from $Z$ to $[x, a]$ is injective: if $m(z)=m\left(z^{\prime}\right)=m$ for distinct $z, z^{\prime} \in Z$ then both $m$ and $y$ are medians for the triple $a, z, z^{\prime}$. The finiteness of $Z$ follows now from the finiteness of $[x, a]$.

Median graphs are instances of median algebras, which can be described as interval structures that enjoy a tripod-like condition. Median algebras can also be defined as sets with a ternary operation $x, y, z \mapsto m(x, y, z)$, called the median operation, that satisfies certain axioms. The latter point of view is adopted in [1] and [10, which we suggest as supplements to our brief presentation.

Definition 3.3 A median algebra is a set $X$ with an interval assignment $(x, y) \mapsto[x, y]$, mapping pairs of points in $X$ to subsets of $X$, so that for all $x, y, z \in X$ the following are satisfied:

- $[x, x]=\{x\}$

- if $z \in[x, y]$ then $[x, z] \subseteq[x, y]$

- $[x, y],[y, z],[z, x]$ have a unique common point, denoted $m(x, y, z)$ 
A subset $A \subseteq X$ is convex if $[x, y] \subseteq A$ for all $x, y \in A$. A subset $A \subseteq X$ is a halfspace if both $A$ and $A^{c}=X \backslash A$ are convex.

A morphism of median algebras is a map $f: X \rightarrow X^{\prime}$ between median algebras that is "betweenness preserving", in the sense that $f([x, y]) \subseteq[f(x), f(y)]$ for all $x, y \in X$.

Definition 3.4 A median algebra is discrete if every interval is finite.

Example 3.5 (Boolean median algebra) Any power set $\mathcal{P}(X)$ is a median algebra under the interval assignment

$$
(A, B) \mapsto[A, B]=\{C: A \cap B \subseteq C \subseteq A \cup B\}
$$

with $(A \cap B) \cup(B \cap C) \cup(C \cap A)=(A \cup B) \cap(B \cup C) \cap(C \cup A)$ being the boolean median of $A, B, C$. The significance of this example is that any median algebra is isomorphic to a subalgebra of a boolean median algebra.

In general, a boolean median algebra $\mathcal{P}(X)$ is far from discrete. We say that $A, B \subseteq X$ are almost equal if their symmetric difference $A \triangle B=(A \backslash B) \cup(B \backslash A)$ is finite; this defines an equivalence relation on $\mathcal{P}(X)$. An almost equality class of $\mathcal{P}(X)$ is a discrete median algebra.

A crucial feature of halfspaces in median algebras is that they separate disjoint convex sets, in the sense that for all disjoint convex sets $C_{1}, C_{2}$ there is a halfspace $A$ so that $C_{1} \subseteq A$ and $C_{2} \subseteq A^{c}$. See [10, §2] for a proof. The walls of a median algebra are pairs of complementary halfspaces, so every two distinct points can be separated by walls. However, the number of walls separating two distinct points may be infinite, as it is the case with $\mathbb{R}$-trees.

We now show that discrete median algebras can be viewed as spaces with walls and, correspondingly, morphisms of discrete median algebras can be viewed as morphisms of spaces with walls (Prop.3.7, equivalence of $a$ ) and $c$ )).

Proposition 3.6 Any discrete median algebra is a space with walls.

Proof We need to show that, for every $x, y \in X$, the set $W(x, y)$ of walls separating $x$ and $y$ is finite. We argue by induction that $|W(x, y)| \leq|[x, y]|-1$.

Suppose $|[x, y]|=2$, i.e., $[x, y]=\{x, y\}$. Assume there are two walls separating $x$ and $y: x \in A, y \in A^{c}$ and $x \in B, y \in B^{c}$. Say $B \nsubseteq A$ and let $z \in B \backslash A$. Then either $m(x, y, z)=x$, yielding $x \in A^{c}$, or $m(x, y, z)=y$, yielding $y \in B$. Both are impossible. 
Suppose $|[x, y]|>2$ and let $z \in[x, y] \backslash\{x, y\}$. Then $[x, z] \cup[z, y] \subseteq[x, y]$ and $[x, z] \cap[z, y]$ consists of a single point, $z$, as $[x, z] \cap[z, y]=[x, z] \cap[z, y] \cap[x, y]$. Using the induction step we obtain:

$$
|W(x, y)| \leq|W(x, z)|+|W(z, y)| \leq|[x, z]|-1+|[z, y]|-1 \leq|[x, y]|-1
$$

Proposition 3.7 Let $f: X \rightarrow X^{\prime}$ be a map, where $X$ and $X^{\prime}$ are median algebras. The following are equivalent:

(a) $f([x, y]) \subseteq[f(x), f(y)]$ for all $x, y \in X$

(b) $f^{-1}\left(C^{\prime}\right)$ is convex in $X$ whenever $C^{\prime}$ is convex in $X^{\prime}$

(c) $f^{-1}\left(A^{\prime}\right)$ is a halfspace in $X$ whenever $A^{\prime}$ is a halfspace in $X^{\prime}$

(d) $f(m(x, y, z))=m(f(x), f(y), f(z))$ for all $x, y, z \in X$

Proof This is a straightforward exercise in median reasoning.

$(\mathrm{a}) \Rightarrow(\mathrm{b})$ : Follows from the definition of convexity.

(b) $\Rightarrow$ (c): Apply (b) to both $A^{\prime}$ and $X^{\prime} \backslash A^{\prime}$.

$(\mathrm{c}) \Rightarrow(\mathrm{d})$ : Assume $f(m(x, y, z)) \neq m(f(x), f(y), f(z))$ for some $x, y, z \in X$. Then $f(m(x, y, z)) \in A^{\prime}$ and $m(f(x), f(y), f(z)) \in X^{\prime} \backslash A^{\prime}$ for some halfspace $A^{\prime}$ in $X^{\prime}$. The latter implies, by the convexity of $A^{\prime}$, that at least two of $\{f(x), f(y), f(z)\}$, say $f(x)$ and $f(y)$, are in $X^{\prime} \backslash A^{\prime}$ i.e. $x, y \in f^{-1}\left(X^{\prime} \backslash A^{\prime}\right)$. Then $m(x, y, z) \in f^{-1}\left(X^{\prime} \backslash A^{\prime}\right)$ as well, which is a contradiction.

$(\mathrm{d}) \Rightarrow(\mathrm{a})$ : Let $z \in[x, y]$, that is $m(x, y, z)=z$. Then $f(z) \in[f(x), f(y)]$ since $m(f(x), f(y), f(z))=f(m(x, y, z))=f(z)$.

The vertex set of a median graph, equipped with the interval structure given by the geodesic intervals, is a discrete median algebra. The converse holds as well: every discrete median algebra arises as the vertex set of a median graph. Thus discrete median algebras are precisely the 0-skeletons of median graphs. This fact, which appears as a special case of our construction (Cor.4.10), relates Chepoi's result [3] that the 1-skeletons of CAT(0) cube complexes are precisely the median graphs, to Roller's result [10, Thm.10.3], that the 0-skeletons of $\mathrm{CAT}(0)$ cube complexes are precisely the discrete median algebras. 


\section{From spaces with walls to median graphs}

We are now ready to prove the main result:

Theorem 4.1 Let $X$ be a space with walls. There exists an injective morphism of spaces with walls

$$
X \stackrel{\sigma}{\longrightarrow} \mathcal{C}^{1}(X)
$$

where $\mathcal{C}^{1}(X)$ is a connected median graph, and $\sigma(X)$ "spans" $\mathcal{C}^{1}(X)$, in the sense that no proper subgraph of $\mathcal{C}^{1}(X)$ containing $\sigma(X)$ is median.

The core idea, which arises naturally in a variety of contexts, can be summarized as follows: given a space, we identify its points with the principal ultrafilters and then we suitably add other ultrafilters. In our case, the suitable ultrafilters are the almost principal ultrafilters. Here are the precise details.

Definition 4.2 Let $X$ be a space with walls. An ultrafilter on $X$ is a nonempty collection $\omega$ of halfspaces that satisfies the following conditions:

- $A \in \omega$ and $A \subseteq B$ imply $B \in \omega$

- either $A \in \omega$ or $A^{c} \in \omega$ but not both

Intuitively, an ultrafilter is a coherent orientation of the walls. Note that every ultrafilter contains $X$. The easiest to single out are the principal ultrafilters, defined for every $x \in X$ to be the collection $\sigma_{x}$ of halfspaces containing $x$.

If $\omega_{1}, \omega_{2}$ are ultrafilters then elements of the symmetric difference $\omega_{1} \Delta \omega_{2}$ come in pairs $\left\{A, A^{c}\right\}$, so we may think of them as being walls. For distinct $x$ and $y$, the set $\sigma_{x} \triangle \sigma_{y}$ describes the walls separating $x$ and $y$, hence it is finite and nonempty.

Consider the graph $\Gamma$ whose vertices are the ultrafilters on $X$, and the edges are defined by: $\omega_{1}$ is adjacent to $\omega_{2}$ if $\frac{1}{2}\left|\omega_{1} \triangle \omega_{2}\right|=1$ i.e. $\omega_{1}$ and $\omega_{2}$ differ by exactly a wall. In general $\Gamma$ is highly disconnected. The connectivity of $\Gamma$ is described in the following statement.

Lemma 4.3 There is a path in $\Gamma$ connecting $\omega_{1}$ and $\omega_{2}$ iff $\omega_{1} \triangle \omega_{2}$ is finite. In fact, the distance between $\omega_{1}$ and $\omega_{2}$ is $\frac{1}{2}\left|\omega_{1} \Delta \omega_{2}\right|$, which is the number of walls by which $\omega_{1}$ and $\omega_{2}$ differ. 
Proof Suppose $\omega_{1}=\theta_{1}, \ldots, \theta_{m+1}=\omega_{2}$ is a path connecting $\omega_{1}$ to $\omega_{2}$. Then

$$
\frac{1}{2}\left|\omega_{1} \triangle \omega_{2}\right|=\frac{1}{2}\left|\left(\theta_{1} \triangle \theta_{2}\right) \triangle \ldots \triangle\left(\theta_{m} \triangle \theta_{m+1}\right)\right| \leq \sum_{i=1}^{m} \frac{1}{2}\left|\theta_{i} \triangle \theta_{i+1}\right|=m
$$

Conversely, suppose $\omega_{1} \triangle \omega_{2}$ is finite. Let $\omega_{1} \triangle \omega_{2}=\left\{A_{1}, \ldots, A_{n}, A_{1}^{c}, \ldots, A_{n}^{c}\right\}$ where $A_{i} \in \omega_{1} \backslash \omega_{2}$ and $A_{i}^{c} \in \omega_{2} \backslash \omega_{1}$. We may assume that each $A_{i}$ is minimal in $\left\{A_{i}, \ldots, A_{n}\right\}$, and we define $\theta_{1}=\omega_{1}, \theta_{i+1}=\theta_{i} \triangle\left\{A_{i}, A_{i}^{c}\right\}$ for $1 \leq i \leq n$. Note that $\theta_{n+1}=\omega_{2}$.

We claim that each $\theta_{i}$ is an ultrafilter. Since $\theta_{i+1}$ is obtained from $\theta_{i}$ by exchanging $A_{i}$ for $A_{i}^{c}$, and since exchanging a minimal halfspace in an ultrafilter for its complement results in an ultrafilter, we are left with showing that $A_{i}$ is minimal in $\theta_{i}$. Suppose there is $B \in \theta_{i}, B \subsetneq A_{i}$. Then $B \notin \omega_{2}$ because $A_{i} \notin \omega_{2}$. As

$$
\theta_{i}=\left(\omega_{1} \backslash\left\{A_{1}, \ldots, A_{i-1}\right\}\right) \cup\left\{A_{1}^{c}, \ldots, A_{i-1}^{c}\right\}
$$

we necessarily have $B \in \omega_{1} \backslash\left\{A_{1}, \ldots, A_{i-1}\right\}$. We obtain $B \in\left\{A_{i}, \ldots, A_{n}\right\}$ which contradicts the fact that $A_{i}$ is minimal in $\left\{A_{i}, \ldots, A_{n}\right\}$.

It follows that the principal ultrafilters lie in the same connected component of $\Gamma$, denoted $\mathcal{C}^{1}(X)$. The vertices of $\mathcal{C}^{1}(X)$ are the ultrafilters $\omega$ for which $\omega \triangle \sigma_{x}$ is finite for some (every) principal ultrafilter $\sigma_{x}$. We call them almost principal ultrafilters.

A helpful description of the geodesic intervals in $\mathcal{C}^{1}(X)$ is the following:

Lemma 4.4 Let $\omega, \omega_{1}, \omega_{2}$ be almost principal ultrafilters. Then:

$$
\omega \in\left[\omega_{1}, \omega_{2}\right] \Leftrightarrow \omega_{1} \cap \omega_{2} \subseteq \omega \Leftrightarrow \omega \subseteq \omega_{1} \cup \omega_{2}
$$

Proof Since $\omega_{1} \Delta \omega$ and $\omega_{2} \Delta \omega$ are finite, we have:

$$
\begin{aligned}
\omega \in\left[\omega_{1}, \omega_{2}\right] & \Leftrightarrow\left|\omega_{1} \triangle \omega\right|+\left|\omega \triangle \omega_{2}\right|=\left|\omega_{1} \triangle \omega_{2}\right|=\left|\left(\omega_{1} \triangle \omega\right) \triangle\left(\omega \triangle \omega_{2}\right)\right| \\
& \Leftrightarrow\left(\omega \triangle \omega_{1}\right) \cap\left(\omega \triangle \omega_{2}\right)=\emptyset \Leftrightarrow \omega_{1} \cap \omega_{2} \subseteq \omega \subseteq \omega_{1} \cup \omega_{2}
\end{aligned}
$$

The equivalence $\omega_{1} \cap \omega_{2} \subseteq \omega \Leftrightarrow \omega \subseteq \omega_{1} \cup \omega_{2}$ holds for arbitrary ultrafilters.

Proposition $4.5 \mathcal{C}^{1}(X)$ is a median graph.

Proof Since geodesic intervals in $\mathcal{C}^{1}(X)$ are of the boolean type described in Example 3.5 the median in $\mathcal{C}^{1}(X)$ of a triple of vertices $\omega_{1}, \omega_{2}, \omega_{3}$, has to be the boolean median

$$
m\left(\omega_{1}, \omega_{2}, \omega_{3}\right)=\left(\omega_{1} \cap \omega_{2}\right) \cup\left(\omega_{2} \cap \omega_{3}\right) \cup\left(\omega_{3} \cap \omega_{1}\right) .
$$


We thus claim that $m\left(\omega_{1}, \omega_{2}, \omega_{3}\right)$ is a vertex in $\mathcal{C}^{1}(X)$. Note that $m\left(\omega_{1}, \omega_{2}, \omega_{3}\right)$ is an ultrafilter whenever $\omega_{1}, \omega_{2}, \omega_{3}$ are ultrafilters. On the other hand, as $\omega_{1} \cap \omega_{2} \subseteq m\left(\omega_{1}, \omega_{2}, \omega_{3}\right) \subseteq \omega_{1} \cup \omega_{2}$, we have that $m\left(\omega_{1}, \omega_{2}, \omega_{3}\right) \triangle \omega_{2} \subseteq \omega_{1} \triangle \omega_{2}$ so $m\left(\omega_{1}, \omega_{2}, \omega_{3}\right) \triangle \omega_{2}$ is finite. Hence $m\left(\omega_{1}, \omega_{2}, \omega_{3}\right)$ is almost principal.

In order to show that the injective map $\sigma: X \rightarrow \mathcal{C}^{1}(X)$ given by $x \mapsto \sigma_{x}$ is the required embedding, we first need to understand the wall structure of $\mathcal{C}^{1}(X)$.

Proposition 4.6 There is a bijective correspondence between the halfspaces of $X$ and the halfspaces of $\mathcal{C}^{1}(X)$ given by

$$
A \mapsto H_{A}=\left\{\omega \in \mathcal{C}^{1}(X): A \in \omega\right\}
$$

Proof Note that the complement of $H_{A}$ is $\mathcal{C}^{1}(X) \backslash H_{A}=H_{A^{c}}$ and each $H_{A}$ is convex: if $\omega \in\left[\omega_{1}, \omega_{2}\right]$ with $\omega_{1}, \omega_{2} \in H_{A}$, then $A \in \omega_{1} \cap \omega_{2} \subseteq \omega$, hence $\omega \in H_{A}$. Thus $H_{A}$ is a halfspace in $\mathcal{C}^{1}(X)$ for every halfspace $A$ in $X$, which shows that the map is well-defined.

The map is injective since $\sigma_{x}$ is in $H_{A}$ iff $x \in A$, i.e., $\sigma^{-1}\left(H_{A}\right)=A$.

We show that the map is surjective. Note that $H_{\emptyset}=\emptyset$ and $H_{X}=\mathcal{C}^{1}(X)$. Let $H$ be a proper halfspace in $\mathcal{C}^{1}(X)$. Pick $\omega \in H, \omega^{\prime} \notin H$ and consider a path $\omega=\omega_{0}, \ldots, \omega_{n}=\omega^{\prime}$ connecting them. Then $H$ cuts an edge in the path, in the sense that $\omega_{i} \in H$ and $\omega_{i+1} \notin H$ for some $i$. Suppose the edge $\omega_{i} \omega_{i+1}$ is obtained by exchanging $A \in \omega_{i}$ for $A^{c} \in \omega_{i+1}$. We claim that $H=H_{A}$. If $\omega \in H_{A}$, that is $A \in \omega$, then $\omega_{i} \subseteq \omega_{i+1} \cup \omega$, i.e., $\omega_{i} \in\left[\omega_{i+1}, \omega\right]$. We get $\omega \in H$, since otherwise the convexity of $\mathcal{C}^{1}(X) \backslash H$ would imply $\omega_{i} \notin H$. Thus $H_{A} \subseteq H$. Similarly $H_{A^{c}} \subseteq \mathcal{C}^{1}(X) \backslash H$, which by complementation becomes $H \subseteq H_{A}$.

We obtain a bijective correspondence between the walls of $X$ and the walls of $\mathcal{C}^{1}(X)$ given by $\left\{A, A^{c}\right\} \mapsto\left\{H_{A}, H_{A^{c}}\right\}$.

Corollary 4.7 On $\mathcal{C}^{1}(X)$, the wall metric and the path metric coincide.

Proof Let $\omega_{1}, \omega_{2}$ be vertices in $\mathcal{C}^{1}(X)$. The wall metric counts the walls $\left\{H_{A}, H_{A^{c}}\right\}$ in $\mathcal{C}^{1}(X)$ separating $\omega_{1}, \omega_{2}$. A wall $\left\{H_{A}, H_{A^{c}}\right\}$ separates $\omega_{1}, \omega_{2}$ iff $\left\{A, A^{c}\right\} \in \omega_{1} \triangle \omega_{2}$. The path metric counts the walls $\left\{A, A^{c}\right\}$ in $\omega_{1} \triangle \omega_{2}$.

Proposition 4.8 The map $\sigma: X \rightarrow \mathcal{C}^{1}(X)$ given by $x \mapsto \sigma_{x}$ is an injective morphism of spaces with walls, and an isometric embedding when $X$ is equipped with the wall metric. 
Proof $\sigma$ is a morphism of spaces with walls since each halfspace in $\mathcal{C}^{1}(X)$ is of the form $H_{A}$ for some halfspace $A$ in $X$, and $\sigma^{-1}\left(H_{A}\right)=A$. We have that $d_{w}(x, y)=\frac{1}{2}\left|\sigma_{x} \triangle \sigma_{y}\right|$ and the right-hand side is the distance between $\sigma_{x}$ and $\sigma_{y}$ in $\mathcal{C}^{1}(X)$.

Proposition 4.9 As a discrete median algebra, $\mathcal{C}^{1}(X)$ is generated by the principal ultrafilters $\left\{\sigma_{x}: x \in X\right\}$.

Proof Let $M \subseteq \mathcal{C}^{1}(X)$ be the median subalgebra generated by $\left\{\sigma_{x}: x \in X\right\}$. We proceed by contamination, assuming that $\omega \in M$ and $\omega \omega^{\prime}$ is an edge in $\mathcal{C}^{1}(X)$, and proving that $\omega^{\prime} \in M$. Suppose the edge $\omega \omega^{\prime}$ is obtained by exchanging $A^{c} \in \omega$ for $A \in \omega^{\prime}$. Let $\zeta \in M \cap H_{A}$ be closest to $\omega$. See Figure 2,

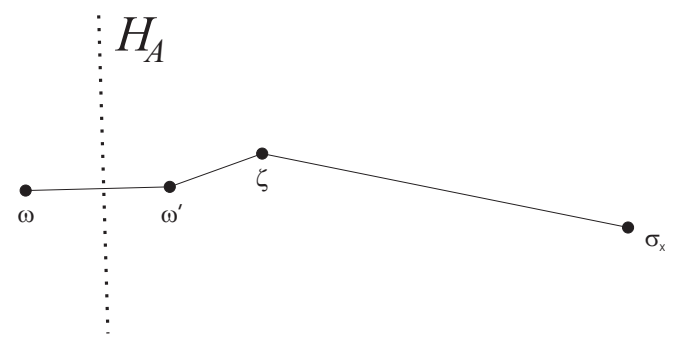

Figure 2: The principal ultrafilters span

We claim that $\zeta=\omega^{\prime}$. First, note that $\zeta \in\left[\omega, \sigma_{x}\right]$ for all $x \in A$, since otherwise $m\left(\omega, \zeta, \sigma_{x}\right)$ would be closer to $\omega$. Second, $\omega^{\prime} \in[\omega, \zeta]$. Hence $\zeta \in\left[\omega^{\prime}, \sigma_{x}\right]$, i.e., $\zeta \subseteq \omega^{\prime} \cup \sigma_{x}$ for all $x \in A$. If there is $B \in \zeta \backslash \omega^{\prime}$ then $B \in \sigma_{x}$ for all $x \in A$, so $A \subseteq B$ and hence $B \in \omega^{\prime}$, which is a contradiction. Thus $\zeta \subseteq \omega^{\prime}$, so $\zeta=\omega^{\prime}$.

In particular, any discrete median algebra is isomorphic to a median graph.

Corollary 4.10 If $X$ is a discrete median algebra then $\sigma: X \rightarrow \mathcal{C}^{1}(X)$ is a median isomorphism.

Proof One checks directly that $m\left(\sigma_{x}, \sigma_{y}, \sigma_{z}\right)=\sigma_{m(x, y, z)}$. As $\left\{\sigma_{x}: x \in X\right\}$ is closed under the median operation, it equals $\mathcal{C}^{1}(X)$, in other words $\sigma$ is onto. Being a bijective morphism of spaces with walls between median algebras, $\sigma$ is a median isomorphism.

Finally, we consider the problem of extending a group action on $X$ to a group action on $\mathcal{C}^{1}(X)$. 
Proposition 4.11 Given a morphism of spaces with walls $f: X \rightarrow X^{\prime}$, there exists a unique median morphism $f_{*}: \mathcal{C}^{1}(X) \rightarrow \mathcal{C}^{1}\left(X^{\prime}\right)$ such that the following diagram commutes:

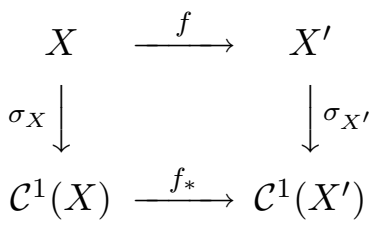

Proof Uniqueness is clear: a median morphism that makes the above diagram commute is determined on $\left\{\sigma_{x}: x \in X\right\}$, which spans $\mathcal{C}^{1}(X)$.

For the existence part, note that the condition $f_{*}\left(\sigma_{x}\right)=\sigma_{f(x)}$ can be expressed as $f_{*}\left(\sigma_{x}\right)=\left\{A^{\prime} \subseteq X^{\prime}\right.$ halfspace $\left.: f^{-1}\left(A^{\prime}\right) \in \sigma_{x}\right\}$. This suggests the following definition:

$$
f_{*}(\omega)=\left\{A^{\prime} \subseteq X^{\prime} \text { halfspace }: f^{-1}\left(A^{\prime}\right) \in \omega\right\}
$$

Let us check that $f_{*}$ is well-defined. First, $f_{*}(\omega)$ is an ultrafilter on $X^{\prime}$ whenever $\omega$ is an ultrafilter on $X$. Second, $f_{*}\left(\omega_{1}\right) \triangle f_{*}\left(\omega_{2}\right)$ is finite whenever $\omega_{1} \triangle \omega_{2}$ is finite, since

$$
f_{*}\left(\omega_{1}\right) \triangle f_{*}\left(\omega_{2}\right)=\left\{A^{\prime} \subseteq X^{\prime} \text { halfspace }: f^{-1}\left(A^{\prime}\right) \in \omega_{1} \triangle \omega_{2}\right\}
$$

and the equation $f^{-1}\left(A^{\prime}\right)=A$, for a given proper halfspace $A$ of $X$, has finitely many solutions: picking $x \in A, y \in A^{c}$, any solution separates $f(x)$ from $f(y)$.

To show that $f_{*}$ is a median morphism, one immediately sees that $f_{*}$ preserves the boolean median. Alternatively, one may show that $f_{*}$ is a morphism of spaces with walls. Indeed, recall that every halfspace in $\mathcal{C}^{1}\left(X^{\prime}\right)$ is of the form $H_{A^{\prime}}=\left\{\omega^{\prime} \in \mathcal{C}^{1}\left(X^{\prime}\right): A^{\prime} \in \omega^{\prime}\right\}$ for some halfspace $A^{\prime}$ in $X^{\prime}$. Then $f_{*}^{-1}\left(H_{A^{\prime}}\right)$ is a halfspace in $\mathcal{C}^{1}(X)$ :

$$
\begin{aligned}
f_{*}^{-1}\left(H_{A^{\prime}}\right) & =\left\{\omega \in \mathcal{C}^{1}(X): f_{*}(\omega) \in H_{A^{\prime}}\right\}=\left\{\omega \in \mathcal{C}^{1}(X): A^{\prime} \in f_{*}(\omega)\right\} \\
& =\left\{\omega \in \mathcal{C}^{1}(X): f^{-1}\left(A^{\prime}\right) \in \omega\right\}=H_{f^{-1}\left(A^{\prime}\right)}
\end{aligned}
$$

Note that $f_{*}$ need not be a graph morphism.

It follows that every automorphism of spaces with walls $f: X \rightarrow X$ has a unique extension to a median automorphism, hence a graph automorphism as well, $f_{*}: \mathcal{C}^{1}(X) \rightarrow \mathcal{C}^{1}(X)$ given by $f_{*}(\omega)=f(\omega)$. Thus a group action on a space with walls $X$ naturally extends to a group action on its 1 -cubulation $\mathcal{C}^{1}(X)$. 


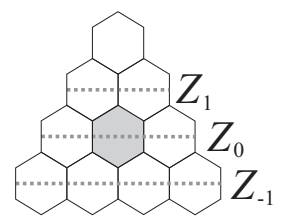

\section{Z-walls}

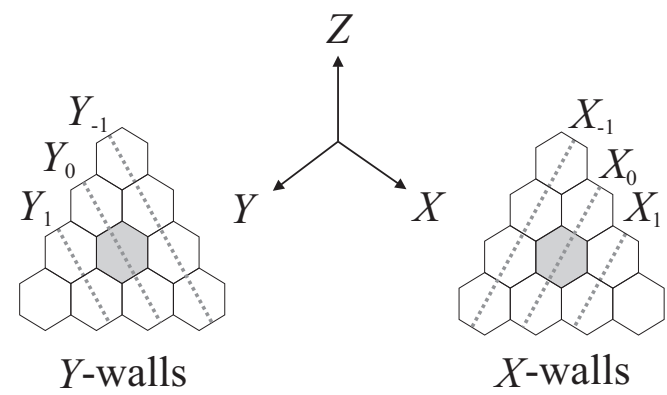

Figure 3: Walls run across three directions

Example 4.12 We cubulate the 1-skeleton of the hexagonal tiling of the plane. The choice of halfspaces is independent along the three directions $X, Y, Z$. But this is also the case with the choice of halfspaces for the 1-skeleton of the usual tiling of $\mathbb{R}^{3}$ by 3 -dimensional cubes. Since this is already a median graph, we conclude that it is the 1-cubulation of the hexagonal tiling of the plane.

\section{References}

[1] H.-J. Bandelt and J. Hedlíková: Median algebras, Discrete Math. 45(1983), 1-30

[2] I.L. Chatterji and G.A. Niblo: From wall spaces to CAT(0) cube complexes, preprint 2003

[3] V. Chepoi: Graphs of some CAT(0) complexes, Adv. Appl. Math. 24(2000), $125-179$

[4] V. Gerasimov: Fixed-point-free actions on cubings, Siberian Adv. Math. 8(1998), no. 3, 36-58

[5] M. Gromov: Hyperbolic groups, Essays in group theory, 75-263, Math. Sci. Res. Inst. Publ., 8, Springer, New York, 1987

[6] F. Haglund and F. Paulin: Simplicité de groupes d'automorphismes d'espaces à courbure négative, The Epstein birthday schrift, Geom. Topol. Monogr. 1(1998) $181-248$ 
[7] G.A. Niblo and M.A. Roller: Groups acting on cubes and Kazhdan's property T, Proc. Amer. Math. Soc. 126(1998), 693-699

[8] G.A. Niblo and L.D. Reeves: Groups acting on CAT(0) cube complexes, Geom. Topol. 1(1997), 1-7

[9] G.A. Niblo and L.D. Reeves: Coxeter groups act on CAT(0) cube complexes, J. Group Theory 6(2003), 399-413

[10] M. Roller: Poc-sets, median algebras and group actions. An extended study of Dunwoody's construction and Sageev's theorem, preprint 1998

[11] M. Sageev: Ends of group pairs and non-positively curved cube complexes, Proc. London Math. Soc. 71(1995), 585-617

[12] D.T. Wise: Cubulating small cancellation groups, preprint 2002

Department of Mathematics, McGill University

Montreal, Canada H3A $2 \mathrm{~K} 6$

Email: bnica@math.mcgill.ca

Received: 31 August 2003 